\title{
Charge response function and a novel plasmon mode in graphene
}

\author{
S. Gangadharaiah, A. M. Farid, and E. G. Mishchenko \\ Department of Physics, University of Utah, Salt Lake City, Utah 84112, USA
}

\begin{abstract}
Polarizability of non-interacting 2D Dirac electrons has a $1 / \sqrt{q v-\omega}$ singularity at the boundary of electron-hole excitations. The screening of this singularity by long-range electron-electron interactions is usually treated within the random phase approximation. The latter is exact only in the limit of $N \rightarrow \infty$, where $N$ is the "color" degeneracy. We find that the ladder-type vertex corrections become crucial close to the threshold as the ratio of the $n$-th order ladder term to the same order RPA contribution is $\ln ^{n}|q v-\omega| / N^{n}$. We perform analytical summation of the infinite series of ladder diagrams which describe excitonic effect. Beyond the threshold, $q v>\omega$, the real part of the polarization operator is found to be positive leading to the appearance of a strong and narrow plasmon resonance.

PACS numbers: $73.23 .-\mathrm{b}, 72.30 .+\mathrm{q}$
\end{abstract}

Introduction. Many properties of interacting twodimensional electrons with linear Dirac spectrum $\epsilon=$ $\pm v p$, found in graphene monolayers, differ sharply from those with the parabolic spectrum present in conventional semiconductor heterostructures [1, 2]. Vanishing density of states at the Dirac point and less effective screening of Coulomb interaction lead to electronic properties of graphene being qualitatively different.

One of the distinct signatures of non-interacting 2D electrons in graphene, caused by the absence of spectrum curvature, is a divergent behavior of the polarization operator (charge susceptibility) at the threshold for the excitation of electron-hole pairs [3, 4, 5, , 6, 7], $\Pi^{(0)}(\omega, q)=-N q^{2} / 16 \sqrt{q^{2} v^{2}-\omega^{2}}$, where $N$ is the degree of degeneracy (in graphene $N=4$ in the absence of magnetic field). The effects of electron-electron interaction on the polarization operator are customarily accounted for by the random phase approximation (RPA) [5, 8, 9, 10], which sums the infinite series of electron loops,

$$
\Pi_{R P A}^{-1}=\left(\Pi^{(0)}\right)^{-1}-V_{q}
$$

where $V_{q}=2 \pi e^{2} / q$, dielectric constant of the substrate being incorporated into the charge $e^{2}$. In particular, RPA predicts that the imaginary part of the polarization operator (which determines absorption in the system and the density-density correlation function) instead of diverging actually vanishes at the threshold according to, $-\Pi_{R P A}^{\prime \prime} \propto g^{-2} \sqrt{\omega-q v}$, where $g=e^{2} / v$ is the dimensionless interaction constant.

A notable property of $2 \mathrm{D}$ electrons, well captured by RPA in a conventional Fermi liquid, is the existence of a low-frequency collective mode of charge oscillations whose spectrum is, $\omega_{q}^{2}=2 e^{2} E_{F} q$, where $E_{F}$ is the Fermi energy. In undoped graphene, $E_{F}=0$, the plasmon mode is thus absent within RPA (finite doping or temperature can lead to the usual RPA plasmons [11, 12]). Mathematically this comes from the fact that the real part of $\Pi^{(0)}$ is negative for $q v>\omega$ (i.e. beyond the domain of electron-hole excitations). In the present paper we demonstrate that this conclusion is an artifact of the approximation (11) and that a collective mode does exist in undoped graphene. This mode describes charge fluctuations in a system of electron-hole pairs interacting in a final state ("excitonic" effect), rather than free pairs, as implied by Eq. (11).

We begin with noting that RPA is much less justified in case of undoped graphene than in the case of conventional parabolic spectrum, where it is formally valid for momenta less than the inverse screening length, $q \ll \kappa$. In undoped graphene the vanishing density of carriers ensures that $\kappa \rightarrow 0$. In particular, direct calculation of the electron self-energy to the second order in interaction demonstrates that non-RPA contributions are generally smaller than the RPA terms only by virtue of $1 / N[13$. The RPA is exact only in the formal limit of $N \rightarrow \infty$, since each RPA term corresponds to the largest number of loops possible in each order of the perturbation theory.

Below we analyze consecutive orders of the perturbation series in the bare interaction $V_{q}=2 \pi e^{2} / q$ and compare RPA diagrams to the ladder corrections. We find that the ratio of the $n$-th ladder term to the corresponding RPA contribution of the same order is $N^{-n} \ln ^{n}\left(\frac{q v}{|q v-\omega|}\right)$ and large close to the threshold. After extracting the leading singular terms in each order, we calculate the infinite ladder series and obtain a non-perturbative expression for the polarization operator near the threshold. Summation of the leading divergencies is possible once we realize that for $q v /|q v-\omega| \gg 1$ the main contribution arises from the processes that involve quasi-one-dimensional motion of interacting particles along the direction of the external momentum $\vec{q}$. In general, when $N \sim \ln \left(\frac{q v}{|q v-\omega|}\right)$, the perturbation expansion of the polarization operator receives similar contributions from both the ladder and RPA terms. Thus, the polarization operator is composed of all possible combinations of ladders and loops.

Non-interacting electrons. To demonstrate our method and to introduce some notations it is instructive to begin with reproducing the zeroth-order polarization operator. 
Close to the resonance, when $|\omega-q v| \ll q v$, only those transitions are important that have momenta $\mathbf{p}$ and $\mathbf{q}$ directed almost along the same line. More precisely, the electron is taken from the state in the lower cone with the momentum $\mathbf{p}$ almost antiparallel to $\mathbf{q}$ and placed in the upper cone in the state with momentum $\mathbf{p}+\mathbf{q}$ which is almost parallel to q. Obviously, this is possible only as long as $p<q$. The relevance of these particular transitions is understood from the form of the polarization operator,

$$
\Pi^{(0)}(\omega, q)=-i N \operatorname{Tr} \sum_{\mathbf{p}} \int \frac{d \epsilon}{2 \pi} \hat{G}_{\epsilon+\omega, \mathbf{p}+\mathbf{q}} \hat{G}_{\epsilon \mathbf{p}},
$$

where the electron Green's function consists of the contributions from both cones $\beta= \pm 1$,

$$
\hat{G}_{\epsilon \mathbf{p}}=\frac{1}{2} \sum_{\beta= \pm 1} \frac{1+\beta \hat{\sigma}_{\mathbf{p}}}{\epsilon-\beta v p+i \beta \eta}
$$

where $\hat{\sigma}_{\mathbf{p}}=\hat{\boldsymbol{\sigma}} \cdot \mathbf{p} / p$ is the projection of the pseudospin Pauli matrix onto the direction of electron momentum. The energy integral in Eq. (2) yields,

$$
\Pi^{(0)}(\omega, q)=\frac{N}{4} \sum_{\beta} \sum_{\mathbf{p}} \frac{\operatorname{Tr}\left(1+\beta \hat{\sigma}_{\mathbf{p}+\mathbf{q}}\right)\left(1-\beta \hat{\sigma}_{\mathbf{p}}\right)}{\omega-\beta v(p+|\mathbf{p}+\mathbf{q}|)+i \beta \eta},
$$

we observe that i) for positive $\omega$ the singular denominator appears for $\beta=+1$, ii) the trace operation in the numerator imposes that $\mathbf{p}$ and $\mathbf{p}+\mathbf{q}$ are antiparallel (for the parallel configuration the trace vanishes). Close to the threshold $(\omega=q v)$ collinear processes become dominant allowing us to approximate $|\mathbf{p}+\mathbf{q}| \approx$ $q-p+\frac{p q \theta^{2}}{2(q-p)}$, where $\theta$ denotes the angle between $\mathbf{p}$ and $-\mathbf{q}$ and $\operatorname{Tr}\left(1+\hat{\sigma}_{\mathbf{p}+\mathbf{q}}\right)\left(1-\hat{\sigma}_{\mathbf{p}}\right) \approx 4$, thus we obtain from Eq. (4),

$$
\Pi^{(0)}(\omega, q)=N \int_{0}^{q} \frac{p d p}{(2 \pi)^{2}} \int_{-\infty}^{\infty} \frac{d \theta}{\omega-q v-v \frac{p q \theta^{2}}{2(q-p)}+i \eta} .
$$

Upon calculating the integrals we recover $\Pi^{(0)}(\omega, q)=$ $-\frac{N}{16 \sqrt{2} v} q^{3 / 2} / \sqrt{q v-\omega}$. Note that, as expected, the relevant angles are small $\theta \sim \sqrt{|1-\omega / q v|} \ll 1$.

First-order correction. To the first order in the electron-electron interaction there are three diagrams, Fig. 1. Of these diagrams, the self-energy correction, Fig. 1a), is the most singular near the threshold,

$$
\Pi_{S E}^{(1)}(\omega, q)=-\frac{N g \sqrt{v}}{16^{2} \sqrt{2}} \frac{q^{5 / 2}}{(q v-\omega)^{3 / 2}} \ln (\mathcal{K} / q),
$$

where $\mathcal{K}$ is the upper momentum cut-off. This singularity indicates that the infinite summation of the self-energy diagrams have to be performed first, thus yielding the renormalization of the electron spectrum. To the lowest order in interaction this gives $v_{p}=v\left[1+\frac{g}{4} \ln (\mathcal{K} / p)\right]$

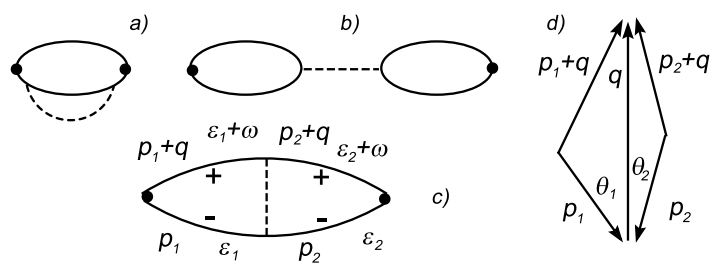

FIG. 1: First-order interaction correction: self-energy correction a), sub-leading RPA correction b), leading vertex correction c). Figure d) illustrates the origin of the singular behavior, $\sim(q v-\omega)^{-1} \ln |q v-\omega|$ from almost collinear electron propagation. Both states $\mathbf{p}_{1}, \mathbf{p}_{2}$ belong to the lower $(-)$ cone, while $\mathbf{p}_{1}+\mathbf{q}, \mathbf{p}_{2}+\mathbf{q}$ correspond to the upper $(+)$ cone.

[4]. In the case of a conventional Fermi liquid the corresponding velocity renormalization is trivial. In case of a linear spectrum considered here, however, logarithmic terms lead to a slight curvature of electron spectrum which can smear the threshold singularities. In what follows we assume the renormalized velocity but neglect this smearing by taking the velocity at a typical momentum, determined by the external momentum $v \equiv v_{q}$.

The next term, 1b), is the RPA-correction which can be readily written as,

$$
\Pi_{R P A}^{(1)}(\omega, q)=\frac{\pi N^{2} g}{16^{2}} \frac{q^{2}}{q v-\omega} .
$$

It can now be verified that the vertex correction, Fig. 1c), yields a contribution which is opposite in sign and more singular than Eq. (7) as a result of the longrange character of Coulomb interaction. The energy integrations are performed independently in each half of the diagram, resulting in

$$
\begin{aligned}
\Pi_{V}^{(1)}(\omega, q)= & -\frac{N}{16} \operatorname{Tr} \sum_{\mathbf{p}_{1} \mathbf{p}_{2}} \sum_{\alpha \beta} \frac{\beta\left(1-\beta \sigma_{\mathbf{p}_{1}+q}\right)\left(1+\beta \sigma_{\mathbf{p}_{1}}\right)}{\omega+\beta v\left(p_{1}+\left|\mathbf{p}_{1}+\mathbf{q}\right|\right)} \\
& \times \frac{\alpha\left(1+\alpha \sigma_{\mathbf{p}_{2}}\right)\left(1-\alpha \sigma_{\mathbf{p}_{2}+\mathbf{q}}\right)}{\omega+\alpha v\left(p_{2}+\left|\mathbf{p}_{2}+\mathbf{q}\right|\right)} V_{\mathbf{p}_{1}-\mathbf{p}_{2}} .
\end{aligned}
$$

To extract the leading contribution we note that it comes from almost collinear processes with $\alpha=\beta=+1$ and both $\mathbf{p}+\mathbf{q}, \mathbf{p}^{\prime}+\mathbf{q}$ are almost parallel to $\mathbf{q}$ and almost antiparallel to both $\mathbf{p}, \mathbf{p}^{\prime}$. Using the small-angle expansion introduced in the previous section, and approximating $V_{\mathbf{p}-\mathbf{p}^{\prime}} \approx e^{2} /\left|p-p^{\prime}\right|$ we obtain

$$
\Pi_{V}^{(1)}(\omega, q)=-\frac{N e^{2}}{(2 \pi)^{4}} \int \frac{1}{\left|p_{1}-p_{2}\right|} \prod_{i=1}^{2} \frac{p_{i} d p_{i} d \theta_{i}}{\omega-q v-v \frac{p_{i} q \theta_{i}^{2}}{2\left(q-p_{i}\right)}} .
$$

The integrals over the two angles are independent and yield the same resonant denominator as in Eq. (7). However, the integrals over the magnitude of electron momenta contain a logarithmic divergence which is due to the long-range nature of Coulomb interaction. To cutoff this divergence we note that, $\left|\mathbf{p}_{1}-\mathbf{p}_{2}\right| \approx\left|p_{1}-p_{2}\right|+$ 
$\frac{p_{1} p_{2} \theta_{12}^{2}}{2\left|p_{1}-p_{2}\right|}$, where $\theta_{12}$ is the angle between two momenta. Recalling that relevant angles $\theta_{12}^{2} \sim|q v / \omega-1|$ and that typical $p_{i} \sim q$, we observe that small-angle expansion fails for $\left|p_{1}-p_{2}\right| \sim q \sqrt{|q v / \omega-1|}$, which should be used as the lower cut-off. As a result we obtain,

$$
\Pi_{V}^{(1)}(\omega, q)=\frac{g N}{24 \pi} \frac{q^{2}}{\omega-q v} \ln \left(\frac{q v}{|\omega-q v|}\right) .
$$

We, therefore, find that RPA loops exceed the vertex terms only when $N \gg \ln \left(\frac{q v}{|\omega-q v|}\right)$. In the opposite limit, close to the resonance, $N \ll \ln \left(\frac{q v}{|\omega-q v|}\right)$ the ladder diagrams dominate. We now turn to the summation of the ladders series.

Summation of the infinite ladder. In the second order in electron-electron interaction the most dominant contribution again comes from the ladder diagram, Fig. 2a), with three pairs of electron lines each yielding $1 / \sqrt{q v-\omega}$-singularity, and two Coulomb interactions providing two additional powers of the logarithm. The other diagrams are less singular at $\omega=q v$. Diagram $2 \mathrm{~b}$ ) requires that at least one of the interaction lines carries large momentum, $\sim q$, while both momenta in the ladder-type diagram 2a) are small. Exchange-type contributions, 2c)-e), though providing the main power-law singularity, involve transferred momenta which are $\sim q$ and lack the additional logarithms (while also lacking extra factors $N$ ). Repeating the arguments from the pre-

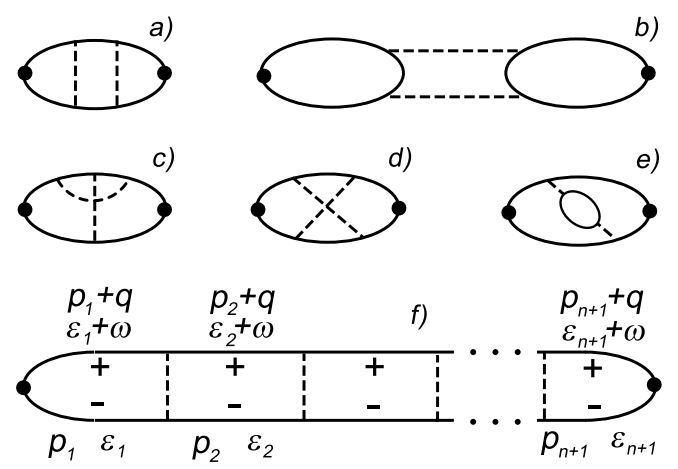

FIG. 2: Leading, a), and subleading, b)-e), non-RPA diagrams of the second order. The ladder diagram f) explains the origin of the most singular, $(q v-\omega)^{-n} \ln ^{n}|q v-\omega|$ contribution of the arbitrary order $n$. All states propagating along the upper/lower part of the ladder belong to the upper/lower cone.

ceding section we realize that to each order in interaction the leading contribution comes from the ladder diagram, Fig. 2f),

$$
\begin{aligned}
& \Pi_{V}^{(n)}(\omega, q)=-N \operatorname{Tr} \int\left[\prod_{i=1}^{n+1} \frac{d \epsilon_{i}}{2 \pi} d \mathbf{p}_{i}\right] V_{\mathbf{p}_{1}-\mathbf{p}_{2} \ldots V_{\mathbf{p}_{n}-\mathbf{p}_{n+1}}} \\
& \times i^{n+1} \hat{G}_{\epsilon_{1}+\omega \mathbf{p}_{1}+\mathbf{q}} \ldots \hat{G}_{\epsilon_{n+1}+\omega \mathbf{p}_{n+1}+\mathbf{q}} \hat{G}_{\epsilon_{n+1} \mathbf{p}_{n+1} \ldots \hat{G}_{\epsilon_{1} \mathbf{p}_{1}}} .
\end{aligned}
$$

All the momenta are almost collinear, with those propagating along the lower part of the ladder, $\mathbf{p}_{i}$, being antiparallel to external wavevector $\mathbf{q}$ and to all the momenta $\mathbf{p}_{i}+\mathbf{q}$ along the upper part of the ladder. Energy integrations are independent in each step and result in the singularity to be the strongest when all the propagators in upper/lower parts of the ladder belong to the upper(+)/lower(-) cones. The subsequent trace operation is easily performed as

$$
\begin{array}{r}
\operatorname{Tr}\left(1-\sigma_{\mathbf{p}_{1}}\right) \ldots\left(1-\sigma_{\mathbf{p}_{n+1}}\right)\left(1+\sigma_{\mathbf{p}_{n+1}+\mathbf{q}}\right) \ldots \\
\ldots \times\left(1+\sigma_{\mathbf{p}_{1}+\mathbf{q}}\right) \approx 4^{n+1}
\end{array}
$$

yielding the $n$-th order contribution to the polarization operator in the form

$$
\begin{aligned}
\Pi_{V}^{(n)}(\omega, q)= & (-1)^{n} N \int V_{p_{1}-p_{2}} \ldots V_{p_{n}-p_{n+1}} \\
& \times \prod_{i=1}^{n+1} \frac{d \mathbf{p}_{i}}{\omega-v\left(p_{i}+\left|\mathbf{p}_{i}+\mathbf{q}\right|\right)+i \eta}
\end{aligned}
$$

Integrating over the angles and then evaluating the integrals over the absolute values of momenta in the leading logarithmic approximation we obtain,

$$
\Pi^{(n)}(\omega, q)=-\frac{1}{8 \sqrt{\pi}} \frac{q^{2}}{\sqrt{2 q v(q v-\omega)}} \frac{\Gamma\left(\frac{3+n}{2}\right)}{\Gamma\left(\frac{4+n}{2}\right)} x^{n},
$$

where we introduced the following dimensionless variable, $x=\frac{g}{2 \sqrt{2}} \sqrt{\frac{q v}{q v-\omega}} \ln \left(\frac{q v}{|q v-\omega|}\right)$. The summation of the infinite series can now be readily performed,

$$
\Pi(\omega, q)=-N q \frac{1+\frac{2}{\pi} \arcsin x-\left(1+\frac{2}{\pi} x\right) \sqrt{1-x^{2}}}{4 v g \ln \left(\frac{q v}{|q v-\omega|}\right) x \sqrt{1-x^{2}}} .
$$

The above result (13) for the polarization ladder is sufficient to calculate the polarization operator in a more general case when $N \sim \ln \left(\frac{q v}{|q v-\omega|}\right) \gg 1$. In this regime both vertex and loop diagrams have to be summed simultaneously. Such summation can be performed by realizing that it yields a geometric series similar to the usual RPA one (1) but with the bare polarization operator $\Pi^{(0)}$ replaced by the polarization ladder $\Pi_{V}$ given by Eq. (13),

$$
\mathcal{P}(\omega, q)=\frac{\Pi_{V}(\omega, q)}{1-V(q) \Pi_{V}(\omega, q)},
$$

Collective mode. Non-zero imaginary part of the polarization operator determines those values of $\omega$ and $q$ for which the dissipation of external field is possible. Imaginary part of Eq. (13), and as a result of Eq. (14), arises in two cases: i) Imaginary $x$ corresponds to the conventional domain, $\omega>q v$, but modified by interaction of electrons and holes. ii) Positive $1<x<\infty$ correspond to the absorption below the threshold and represent a nonperturbative effect which arises from lowering the energy 


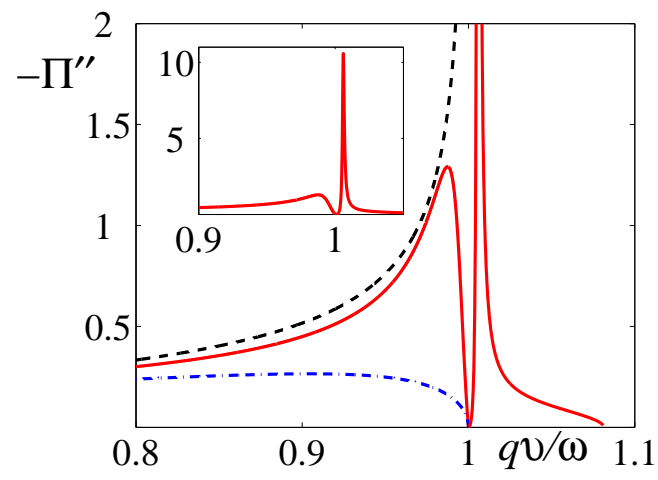

FIG. 3: (Color online) Imaginary part of the polarization operator in units of $q / 4$ for $N=4$ and $g=0.3$ : non-interacting value $\Pi_{0}^{\prime \prime}$ (black dashed line), RPA value $\Pi_{R P A}^{\prime \prime}$ from (11) (blue dot-dashed line) and $\mathcal{P}^{\prime \prime}$ from Eq. (14) that describes collective response of excitons (red solid line). The inset illustrates the relative height of the excitonic plasmon line to the background of individual electron-hole absorption. The position of plasmon $q v=1.006 \omega$, the absorption threshold $q v=1.081 \omega$.

of electron-hole excitations with finite $q$ from attractive Coulomb interaction. The new threshold of absorption $\omega=q u$ is determined from the condition $x=1$ which for weak interactions $g \ll 1$, gives

$$
u=v\left[1-(g \ln g)^{2} / 2\right] .
$$

Even more striking property of Eq. (13) is the behavior of its real part which describes the polarizability of an interacting electron-hole pair. It is easy to see that the real part is positive for $x>1$ [14]. This change in sign, as compared with the real part of the RPA polarizability (1), can already be traced to the first order vertex correction (10), which is opposite to the first order RPA correction (7). This fact has immediate consequence for the collective response of excitons, given by Eq. (14), which develops a pole for a certain value of frequency $\omega(q)$. This new mode describes the propagation of coherent oscillating charge density in a system of interacting electron-hole pairs. The corresponding peak in the absorption has a finite but small width which is due to the fact that the collective mode falls within the range of decay via individual electron-hole pairs. Its behavior is illustrated in Fig. 3 for $N=4$.

Interestingly, the width of the plasmon is maximal around $g \sim 0.3$. For smaller values of the coupling strength the peak narrows by virtue of the narrowing of the whole exciton domain, Eq. (15). For larger values, $g \gg 1$, the peak width decreases as $\propto g^{-2}$ while the height increases only as $\propto g$. The position of the peak in this limit tends to the value determined from the equation (recovered from (13]14) when $x \rightarrow \infty$ ), $\ln \left(\frac{q v}{q v-\omega}\right)=N$, which yields,

$$
\omega(q)=q v\left(1-e^{-N}\right) .
$$

Conclusion. The usual RPA formalism adequately describes dynamical charge fluctuations only in the limit $N \gg \ln \frac{q v}{|q v-\omega|}$. Close to the Dirac cone we find that the $n$-th order term in the ladder series is larger than the corresponding term in the RPA series by a factor of $\ln ^{n}(q v /|q v-\omega|) / N^{n}$. This stronger singularity in the ladder series arises due to the long range interaction between electrons which move almost collinearly to the external momentum $\vec{q}$. The series is summed up analytically yielding a non-perturbative result: the density and spin response functions acquire non-zero imaginary part in the additional frequency range, $q u<\omega<q v$. This extension is a manifestation of the excitonic effect. The reversal of the sign of the electron polarizability in this new domain gives rise to a sharp plasmonic mode which is absent in the conventional RPA. Interestingly, a similar sign reversal of the polarizability due to interactions has been reported to yield a surface plasmon in a different case of a two-dimensional Anderson insulator [15].

Useful discussions with A. Chubukov, M. Raikh and O. Starykh are gratefully acknowledged. This work was supported by DOE, Grant No. DE-FG02-06ER46313.

[1] M. Wilson, Phys. Today 59, 21 (2006).

[2] A. H. Castro Neto, F. Guinea, N. M. R. Peres, K. S. Novoselov, A. K. Geim, arXiv:0709.1163.

[3] K. W. Shung, Phys. Rev. B 34, 1264 (1986).

[4] J. González, F. Guinea, and M.A.H. Vozmediano, Nucl. Phys. B 424, 595 (1994).

[5] J. González, F. Guinea, and M.A.H. Vozmediano, Phys. Rev. B 59, R2474 (1999).

[6] A.V. Chubukov and A.M. Tsvelik, Phys. Rev. B 73, 220503(R) (2006).

[7] D.V. Khveshchenko, Phys. Rev. B 74, 161402(R) (2006).

[8] D.V. Khveshchenko, Phys. Rev. Lett. 87, 246802 (2001).

[9] S. Das Sarma, E.H. Hwang, and W-K. Tse, Phys. Rev. B 75, 121406(R) (2007).

[10] Y. Barlas, T. Pereg-Barnea, M. Polini, R. Asgari, and A. H. MacDonald, Phys. Rev. Lett. 98, 236601 (2007).

[11] E.H. Hwang and S. Das Sarma, Phys. Rev. B 75, 205418 (2007).

[12] O. Vafek, Phys. Rev. Lett. 97, 266406 (2006).

[13] E.G. Mishchenko, Phys. Rev. Lett. 98, 216801 (2007).

[14] For $x>1$ the analytical continuation should be performed from $x \in[0,1)$ to $(1, \infty)$. From the requirement that $\Pi^{\prime \prime}$ is negative we observe that it should yield $\sqrt{1-x^{2}} \rightarrow-i \sqrt{x^{2}-1}$. This implies that the analytical continuation must be performed in the upper half-plane of complex $x$, so that $\arcsin x \rightarrow \pi / 2+i \ln \left(x+\sqrt{x^{2}-1}\right)$.

[15] T.V. Shahbazyan and M.E. Raikh, Phys. Rev. B 53, 7299 (1996). 